\title{
A $q$-BETA INTEGRAL ON THE UNIT CIRCLE AND SOME BIORTHOGONAL RATIONAL FUNCTIONS
}

\author{
WALEED A. AL-SALAM AND MOURAD E. H. ISMAIL
}

(Communicated by Hal L. Smith)

\begin{abstract}
In this paper we first consider a pair of polynomial sets which are biorthogonal on the unit circle with respect to a complex weight function. We then show how the biorthogonality of this pair of polynomial sets implies a $q$-beta integral which in turn leads to a pair of biorthogonal rational functions. Finally we show that the asymptotics for these pairs of rational functions exhibit qualitative properties reminiscent of the Szegö theory for orthogonal polynomials.
\end{abstract}

\section{INTRODUCTION}

When Askey edited the collected papers of Szegö [18] he inserted some very interesting commentaries. In one of these [17] he introduced a set of polynomials orthogonal on the unit circle. They are

$$
S_{n}^{a}(z)=\sum_{k=0}^{n} \frac{\left(a q^{2} ; q^{2}\right)_{k}\left(a ; q^{2}\right)_{n-k}}{\left(q^{2} ; q^{2}\right)_{k}\left(q^{2} ; q^{2}\right)_{n-k}}(z / q)^{k},
$$

which he showed, assuming $a$ real, to be orthogonal on the unit circle with respect to the weight function

$$
\begin{aligned}
w(\theta) & =\frac{\left(q z, q / z ; q^{2}\right)_{\infty}}{\left(a q z, a q / z ; q^{2}\right)_{\infty}} \\
& =\frac{\left(a q^{2}, a q^{2} ; q^{2}\right)_{\infty}}{\left(q^{2}, a^{2} q^{2} ; q^{2}\right)_{\infty}} \psi_{1}\left(1 / a ; q^{2} a ; q^{2}, q a z\right), \quad z=e^{i \theta} .
\end{aligned}
$$

The case $a=0$ leads to the Szegö polynomials which are the subject of [17].

In [17] Askey remarked that Hahn [9] considered a more general nonpositive weight

$$
f(z)={ }_{1} \psi_{1}\left(1 / a ; q b ; q^{1 / 2} a z\right)=\sum_{k=-\infty}^{\infty} \frac{(1 / a ; q)_{k}}{(q b ; q)_{k}}\left(q^{1 / 2} a z\right)^{k} .
$$

Received by the editors October 1, 1992.

1991 Mathematics Subject Classification. Primary 33D45, 33D05; Secondary 42A65.

Key words and phrases. Biorthogonal rational functions, $q$-beta integrals, asymptotics, generating functions, Askey-Wilson integral.

Reseach of the first author was partially supported by NSERC (Canada) grant A2975.

Research of the second author was partially supported by the National Science Foundation under grant DMS 9203659. 
Hahn mentioned more general polynomials orthogonal on the unit circle with respect to $f(z)$. This assertion, which Hahn stated but did not prove, is false. Baxter [3] showed that if the weight function is not positive and certain Toeplitz determinants do not vanish, then there exists a unique pair of polynomial sets which are biorthogonal on the unit circle. This suggests that the proper setting for Hahn's result lies in Baxter's work. Indeed Pastro [14] did that and more. He started from a $q$-beta integral (equivalent to one of Ramanujan's) and introduced a pair of polynomial sets which are biorthogonal on the unit circle and more general than (1.1) and whose weight function is equivalent to the one that Hahn considered.

In this work we first reconsider Pastro's biorthogonal polynomials and show, using generating relations, that they imply a $q$-beta integral which is more general than (2.1) although it is less general than the one obtained, using a different method, by Gasper (see [6, (4.11.4)]).

In $\S 3$ we show how this $q$-beta integral leads to a pair of four-parameter rational functions which are biorthogonal on the unit circle. This seems to be the first system of biorthogonal rational functions, on the unit circle, with so many free parameters and seems to be an analog of the Askey-Wilson polynomials on the unit circle. For related systems of rational functions biorthogonal on subsets of the real line we refer the interested reader to $[1,10,11,15,16]$. One may also consult the recent monograph [5] for applications to numerical analysis and the interesting work of Iserles and Norsett [10] for a general set-up for the concept of biorthogonality. In $\S 3$ we also consider the asymptotics of these biorthogonal polynomials and functions. Also we obtain results which suggest that there are analogies for rational functions biorthogonal on the unit circle with corresponding results for polynomials orthogonal on the real line due to Szegö [7, 8] and results for polynomial sets biorthogonal on the unit circle due to Baxter [3].

In the following all products are to the base $q$ which we assume to be real and $|q|<1$. We shall also assume that $\left|a q^{1 / 2}\right|<1$ and $\left|b q^{1 / 2}\right|<1$. Furthermore, we use the notation

$$
(a ; q)_{\infty}=\prod_{k=0}^{\infty}\left(1-a q^{k}\right), \quad(a ; q)_{n}=\frac{(a ; q)_{\infty}}{\left(a q^{n} ; q\right)_{\infty}}, \quad n=0, \pm 1, \pm 2, \ldots,
$$

$$
{ }_{r} \phi_{r-1}\left(\begin{array}{c}
a_{1} a_{2}, \ldots, a_{r} ; q, z \\
b_{1}, b_{2}, \ldots, b_{r-1}
\end{array}\right):=\sum_{k=0}^{\infty} \frac{\left(a_{1} ; q\right)_{k}\left(a_{2} ; q\right)_{k} \cdots\left(a_{r} ; q\right)_{k}}{(q ; q)_{k}\left(b_{1} ; q\right)_{k}\left(b_{2} ; q\right)_{k} \cdots\left(b_{r-1} ; q\right)_{k}} z^{k}
$$

and

$$
1 \psi_{1}(\alpha ; \beta ; q, z):=\sum_{k=-\infty}^{\infty} \frac{(\alpha ; q)_{k}}{(\beta ; q)_{k}}, \quad\left|\frac{\beta}{\alpha}\right|<|z|<1
$$

For compactness we shall use the notation $(a)_{n}$ to mean $(a ; q)_{n}$ and $(a)_{\infty}$ for $(a ; q)_{\infty}$ if the base $q$ is used. We shall also use the contracted notation

$$
\left(a_{1}, a_{2}, \ldots, a_{n} ; q\right)_{N}=\prod_{k=1}^{n}\left(a_{k} ; q\right)_{N}, \quad \text { where } N \text { is finite or infinite }
$$


Furthermore, $\bar{z}$ shall denote the complex conjugate of $z$, and if $f(z)$ is a power series in $z$, then $\bar{f}(z)$ is the power series whose coefficients are the conjugates of the power series coefficients of $f$. For a polynomial $P(z)$ of degree $n$ we use the standard notation $P^{*}(z)=z^{n} \bar{P}(1 / z)$.

2. Biorthogonal POLYNOMials AND A $Q$-BETA INTEGRAL

Pastro [14] started with a $q$-beta integral of Ramanujan and showed that it is equivalent to

$$
\frac{1}{2 \pi} \int_{0}^{2 \pi} \frac{\left(q^{1 / 2} z, q^{1 / 2} / z ; q\right)_{\infty}}{\left(a q^{1 / 2} z, b q^{1 / 2} / z ; q\right)_{\infty}}=\frac{(q a, q b ; q)_{\infty}}{(q, a b q ; q)_{\infty}}
$$

which suggested to him to use, as a weight function, the function

$$
\Omega(z)={ }_{1} \psi_{1}\left(1 / a ; q b ; q, q^{1 / 2} a z\right)=\frac{\left(q, a b q, q^{1 / 2} z, q^{1 / 2} / z ; q\right)_{\infty}}{\left(a q, b q, q^{1 / 2} a z, q^{1 / 2} b / z ; q\right)_{\infty}}
$$

where $\left|b q^{1 / 2}\right|<|z|<\left|a q^{1 / 2}\right|^{-1}$ so that the case $b=a=\bar{a}$ (i.e., $a$ and $b$ are equal and real) gives the weight, $w(\theta)$, used by Askey in his remarks. The last equality in (2.2) is the sum of a general ${ }_{1} \psi_{1}$ (see [6, p. 126]).

The function $\Omega(z)$ is complex on the unit circle when $a \neq \bar{b}$, and thus according to Baxter [3] we are assured of the existence of a unique pair of polynomial sets which are biorthogonal on the unit circle when certain Toeplitz determinants do not vanish.

Pastro proved that the following pair of polynomial sets are biorthogonal on the unit circle:

$$
\begin{aligned}
p_{n}(z)=p_{n}(z, a, b) & =\sum_{k=0}^{n} \frac{(a q)_{k}(b)_{n-k}}{(q)_{k}(q)_{n-k}}\left(q^{-1 / 2} z\right)^{k} \\
& =\frac{(b)_{n}}{(q)_{n}} \sum_{k=0}^{n} \frac{\left(q^{-n}\right)_{k}(a q)_{k}}{(q)_{k}\left(q^{1-n} / b\right)_{k}}\left(q^{1 / 2} z / b\right)^{k}
\end{aligned}
$$

and

$$
q_{n}(z)=q_{n}(z, a, b):=p_{n}(z, \bar{b}, \bar{a}) .
$$

We note that although Pastro assumed the parameters $a$ and $b$ are real, in fact they do not have to be and the biorthogonality with respect to the complex weight (2.2) still holds.

The corresponding monic polynomial sets are given by

$$
\widehat{p}_{n}(z)=q^{n / 2} \frac{(q)_{n}}{(a q)_{n}} p_{n}(z, a, b), \quad \hat{q}_{n}(z)=q^{n / 2} \frac{(q)_{n}}{(\bar{b} q)_{n}} q_{n}(z, a, b) .
$$

By using easy calculations we find the recurrence relations

$$
\hat{p}_{n+1}(z)+z \hat{p}_{n}(z)+\hat{p}_{n+1}(0) \hat{q}_{n}^{*}(z)
$$

and

$$
\hat{q}_{n+1}(z)=z \hat{q}_{n}(z)+\hat{q}_{n+1}(0) \hat{p}_{n}^{*}(z) .
$$

From (2.6) and (2.7) we see that the monic biorthogonal sets satisfy a threeterm recurrence relation of the form

$$
\hat{p}_{n+2}(z)=\left(z+c_{n}\right) \hat{p}_{n+1}(z)-\lambda_{n} z \hat{p}_{n}(z),
$$


where

$$
\begin{gathered}
c_{n}=\frac{\hat{p}_{n+2}(0)}{\hat{p}_{n+1}(0)}=q^{1 / 2} \frac{1-b q^{n+1}}{1-a q^{n+2}}, \\
\lambda_{n}=c_{n}\left(1-\hat{p}_{n+1}(0) \overline{\hat{q}_{n+1}(0)}\right) \\
=c_{n}\left(1-\frac{(1-a)(1-b) q^{n+1}}{\left(1-a q^{n+1}\right)\left(1-b q^{n+1}\right)}\right) .
\end{gathered}
$$

The polynomial set $\left\{\hat{q}_{n}(z)\right\}$ satisfies a similar recurrence relation.

The orthgonality relation becomes

$$
\frac{1}{2 \pi} \int_{0}^{2 \pi} \Omega\left(e^{i \theta}\right) p_{n}\left(e^{i \theta}\right) \overline{q_{m}\left(e^{i \theta}\right)} d \theta=\frac{(a b q)_{n}}{(q)_{n}} q^{-n} \delta_{m n}
$$

We now proceed to recast (2.11) in terms of generating functions. Using (2.3) and the $q$-binomial theorem (see $[6,($ II.8)]), we can easily derive the generating relations

$$
G(z, t)=\sum_{n=0}^{\infty} p_{n}(z) t^{n}=\frac{\left(b t, a q^{1 / 2} z t ; q\right)_{\infty}}{\left(t, q^{-1 / 2} z t ; q\right)_{\infty}}
$$

and

$$
H(z, t)=\sum_{n=0}^{\infty} q_{n}(z) t^{n}=\frac{\left(\bar{a} t, \bar{a} q^{1 / 2} t z ; q\right)_{\infty}}{\left(t, q^{-1 / 2} z t ; q\right)_{\infty}} .
$$

Now calculating the integral using the above generating relations and the biorthogonality relation $(2.11)$ we obtain

$$
\frac{1}{2 \pi} \int_{0}^{2 \pi} \Omega\left(e^{i \theta}\right) G\left(e^{i \theta}, t_{2}\right) \overline{H\left(e^{i \theta}, \bar{t}_{1}\right)} d \theta=\sum_{n=0}^{\infty} t_{1}^{n} t_{2}^{n} \frac{(a b q)_{n}}{(q)_{n}} q^{-n}=\frac{\left(t_{1} t_{2} a b\right)_{\infty}}{\left(t_{1} t_{2} / q\right)_{\infty}} .
$$

On the other hand using the right-hand side of (2.12) and (2.15) the integral in (2.14) becomes

$$
\frac{1}{2 \pi} \int_{0}^{2 \pi} \Omega\left(e^{i \theta}\right) \frac{\left(a t_{1}, a q^{1 / 2} t_{1} / z ; q\right)_{\infty}\left(b t_{2}, a q^{1 / 2} t_{2} z ; q\right)_{\infty}}{\left(t_{1}, q^{-1 / 2} t_{1} / z ; q\right)_{\infty}\left(t_{2}, q^{-1 / 2} t_{2} z ; q\right)_{\infty}} d \theta
$$

Thus (2.14) and (2.15) lead to a $q$-beta integral, which we state as

Theorem 1 ( $q$-beta integral). The following q-beta integral holds when $\left|a q^{1 / 2}\right|<$ $1,\left|b q^{1 / 2}\right|<1,\left|t_{1}\right|<\left|q^{1 / 2}\right|$, and $\left|t_{2}\right|<\left|q^{1 / 2}\right|$ :

$$
\begin{gathered}
\frac{1}{2 \pi} \int_{0}^{2 \pi} \frac{\left(q^{1 / 2} z, q^{1 / 2} / z, a q^{1 / 2} t_{2} z, b q^{1 / 2} t_{1} / z ; q\right)_{\infty}}{\left(a q^{1 / 2} z, b q^{1 / 2} / z, q^{-1 / 2} t_{2} z, q^{-1 / 2} t_{1} / z ; q\right)_{\infty}} d \theta \\
=\frac{\left(a q, b q, t_{1}, t_{2}, a b t_{1} t_{2} ; q\right)_{\infty}}{\left(q, a b q, a t_{1}, b t_{2}, t_{1} t_{2} q^{-1}\right)_{\infty}} \quad\left(z=e^{i \theta}\right)
\end{gathered}
$$

Note that the case $t_{1}=t_{2}=0$ yields the $q$-beta integral $(2.1)$.

\section{BIORTHOgONAL RATIONAL FUNCTIONS}

In this section we shall apply (2.16) to introduce a pair of rational functions biorthogonal on the unit circle. To do this, we first rewrite $(2.16)$ in the form

$$
\frac{1}{2 \pi i} \int_{|z|=1} K(z) \frac{d z}{z}=1
$$


where

$$
\begin{aligned}
K(z) & =K\left(z, t_{1}, t_{2}\right) \\
& :=\frac{\left(q^{1 / 2} z, q^{1 / 2} / z, a t_{2} q^{1 / 2} z, b t_{1} q^{1 / 2} / z, q, q b q, a t_{1}, b t_{2}, t_{1} t_{2} q^{-1} ; q\right)_{\infty}}{\left(a q^{1 / 2} z, b q^{1 / 2} / z, q^{-1 / 2} t_{2} z, q^{-1 / 2} t_{1} / z, a q, b q, t_{1}, t_{2}, a b t_{1} t_{2} ; q\right)_{\infty}}
\end{aligned}
$$

Note that $(3.1)$ is valid for all values of the parameters $t_{1}, t_{2}$ such that $\left|t_{1}\right|<$ $\left|q^{1 / 2}\right|$, and $\left|t_{2}\right|<\left|q^{1 / 2}\right|$.

The functional relationship

$$
\begin{aligned}
K(z, & \left.t_{1}, t_{2}\right) \frac{\left(q^{-1 / 2} t_{1} / z\right)_{m}\left(q^{-1 / 2} t_{2} z\right)_{k}}{\left(b q^{1 / 2} t_{1} / z\right)_{m}\left(a q^{1 / 2} t_{2} z\right)_{k}} \\
& =K\left(z, t_{1}^{m}, t_{2} q^{k}\right) \frac{\left(a t_{1}\right)_{m}\left(b t_{2}\right)_{k}\left(t_{1} t_{2} q^{-1}\right)_{m+k}}{\left(t_{1}\right)_{m}\left(t_{2}\right)_{k}\left(a b t_{1} t_{2} q^{-1}\right)_{m+k}}
\end{aligned}
$$

establishes the following evaluation of a $q$-beta integral:

$$
\frac{1}{2 \pi} \int_{0}^{2 \pi} K\left(e^{i \theta}\right) \frac{\left(q^{-1 / 2} t_{1} e^{-i \theta}\right)_{m}\left(q^{-1 / 2} t_{2} e^{i \theta}\right)_{k}}{\left(b q^{1 / 2} t_{1} e^{-i \theta}\right)_{m}\left(a q^{1 / 2} t_{2} e^{i \theta}\right)_{k}} d \theta=\frac{\left(a t_{1}\right)_{m}\left(b t_{2}\right)_{k}\left(t_{1} t_{2} q^{-1}\right)_{m+k}}{\left(t_{1}\right)_{m}\left(t_{2}\right)_{k}\left(a b t_{1} t_{2}\right)_{m+k}}
$$

Now multiply both sides of this equation by

$$
\frac{\left(q^{-n}\right)_{k}\left(t_{2}\right)_{k}\left(a b t_{1} t_{2} q^{n-1}\right)_{k}}{(q)_{k}\left(b t_{2}\right)\left(t_{1} t_{2} / q\right)_{k}} q^{k}
$$

Then sum over $k=0,1,2, \ldots, n$ to get

$$
\begin{aligned}
\frac{1}{2 \pi} \int_{0}^{2 \pi} K\left(e^{i \theta}\right) \frac{\left(q^{-1 / 2} t_{1} / z\right)_{m}}{\left(q^{1 / 2} b t_{1} / z\right)_{m}}{ }_{4} \phi_{3}\left(\begin{array}{c}
q^{-n}, q^{-1 / 2} t_{2} z, t_{2}, a b t_{1} t_{2} q^{n-1} ; q, q \\
b t_{2}, a q^{1 / 2} t_{2} z, t_{1} t_{2} q^{-1}
\end{array}\right) d \theta \\
\quad=\frac{\left(a t_{1}\right)_{m}\left(t_{1} t_{2} q^{-1}\right)_{m}}{\left(t_{1}\right)_{m}\left(a b t_{1} t_{2}\right)_{m}} \phi_{2}\left(\begin{array}{c}
q^{-n}, t_{1} t_{2} q^{m-1}, a b t_{1} t_{2} q^{n-1} ; q, q \\
t_{1} t_{2} q^{-1}, a b t_{1} t_{2} q^{m}
\end{array}\right) \\
=\frac{\left(q^{-m}\right)_{n}\left(q^{-n} /(a b)\right)_{n}}{\left(t_{1} t_{2} q^{-1}\right)_{n}\left(\left(a b t_{1} t_{2}\right)^{-1} q^{-1-n-m}\right)_{n}} \cdot \frac{\left(a t_{1}\right)_{m}\left(t_{1} t_{2} q^{-1}\right)_{m}}{\left(t_{1}\right)_{m}\left(a b t_{1} t_{2}\right)_{m}}
\end{aligned}
$$

Let

$$
r_{n}(z)=r_{n}\left(z, a, b, t_{1}, t_{2}\right)={ }_{4} \phi_{3}\left(\begin{array}{c}
q^{-n}, q^{-1 / 2} t_{2} z, t_{2}, a b t_{1} t_{2} q^{n-1} ; q, q \\
a q^{1 / 2} t_{2} z, b t_{2}, t_{1} t_{2} q^{-1}
\end{array}\right)
$$

and

$$
s_{n}(z)=r_{n}\left(z, \bar{b}, \bar{a}, \bar{t}_{2}, \bar{t}_{1}\right)
$$

Then (3.4) shows that

$$
\begin{aligned}
& \frac{1}{2 \pi} \int_{0}^{2 \pi} K\left(e^{i \theta}\right) \overline{s_{m}\left(e^{i \theta}, a, b\right)} r_{n}\left(e^{i \theta}, a, b\right) d \theta \\
& \quad= \begin{cases}0 & \text { if } m=0,1,2, \ldots, n-1, \\
\frac{(a b q)_{n}\left(a b t_{1} t_{2} q^{n-1}\right)_{n}(q)_{n}}{\left(a b t_{1} t_{2}\right)_{2 n}\left(t_{1} t_{2} q^{-1}\right)_{n}}\left(t_{1} t_{2} q^{-1}\right)^{n} & \text { if } m=n .\end{cases}
\end{aligned}
$$

In a similar fashion we can derive a relation similar to (3.7) with $r$ and $s$ interchanged. Thus we obtain the biorthogonality relation in 
Theorem 2. The rational functions $\left\{r_{n}(z)\right\}$ and $\left\{s_{n}(z)\right\}$ satisfy the biorthogonality relation

$$
\frac{1}{2 \pi} \int_{0}^{2 \pi} K\left(e^{i \theta}\right) \overline{s_{m}\left(e^{i \theta}\right)} r_{n}\left(e^{i \theta}\right) d \theta=\frac{(a b q)_{n}\left(a b t_{1} t_{2} q^{n-1}\right)_{n}(q)_{n}}{\left(a b t_{1} t_{2}\right)_{2 n}\left(t_{1} t_{2} q^{-1}\right)_{n}} \cdot\left(t_{1} t_{2} q^{-1}\right)^{n} \delta_{n m} .
$$

Let $\left\{P_{n}(z)\right\}$ and $\left\{Q_{n}(z)\right\}$ be the biorthogonal polynomial sets corresponding to (2.2) and (2.3). To get the asymptotics for these polynomial sets we use (2.3), (2.4), and (2.11) to write

$$
\begin{aligned}
P_{n}(z) & =\left[\frac{(q)_{n} q^{n}}{(a b q)_{n}}\right]^{1 / 2} p_{n}(z) \\
& =\left\{\frac{(q)_{n} q^{n}}{(a b q)_{n}}\right\}^{1 / 2} \sum_{k=0}^{n} \frac{(a q)_{n-k}(b)_{k}}{(q)_{n-k}(q)_{k}}\left(q^{-1 / 2} z\right)^{n-k} \\
& \sim(z)^{n}\left[\frac{(q)_{\infty}}{(a b q)_{\infty}}\right]^{1 / 2} \frac{(a q)_{\infty}}{(q)_{\infty}} \sum_{k=0}^{\infty} \frac{(b)_{k}}{(q)_{k}}\left(q^{1 / 2} / z\right)^{k} \\
& =z^{n}\left[\frac{(a q)_{\infty}(a q)_{\infty}}{(q)_{\infty}(a b q)_{\infty}}\right]^{1 / 2} \frac{\left(q^{1 / 2} b / z\right)_{\infty}}{\left(q^{1 / 2} / z\right)_{\infty}}
\end{aligned}
$$

Similarly we get for $\left\{Q_{n}(z)\right\}$

$$
Q_{n}(z) \sim z^{n}\left[\frac{(\bar{b} q)_{\infty}(\bar{b} q)_{\infty}}{(q)_{\infty}(\bar{a} \bar{b} q)_{\infty}}\right]^{1 / 2} \frac{\left(q^{1 / 2} \bar{a} / z\right)_{\infty}}{\left(q^{1 / 2} / z\right)_{\infty}}
$$

Now we can state a limiting relationship.

Theorem 3. As $n \rightarrow \infty$ and for $\left|q^{1 / 2}\right|<|z|<\left|q^{-1 / 2}\right|$ we have

$$
P_{n}(z) \bar{Q}_{n}(1 / z) \sim 1 / \Omega(z) .
$$

As for the strong asymptotics of the rational functions (3.5) and (3.6) we utilize an asymptotic formula that Ismail and Wilson [13] obtained for the Askey-Wilson polynomials which show

$$
\text { (3.11) }{ }_{4} \phi_{3}\left(\begin{array}{c}
q^{-n}, a b c d q^{n-1}, a z, a / z ; q, q \\
a b, a c, a d
\end{array}\right) \sim\left(\frac{a}{z}\right)^{n} \frac{(a z, b z, c z, d z ; q)_{\infty}}{\left(z^{2}, a b, a c, a d ; q\right)_{\infty}}
$$

when $|z|<1$. We identify (3.5) with (3.11) and obtain for $|z|>q^{1 / 2}$ the asymptotic relationships

$$
r_{n}(z) \sim q^{-n / 2} t_{2}^{n} z^{n} \frac{\left(t_{2}, a q, q^{1 / 2} b / z, q^{-1 / 2} t_{1} / z ; q\right)_{\infty}}{\left(q^{1 / 2} / z, a q^{1 / 2} t_{2} z, b t_{2}, t_{2} t_{1} q^{-1} ; q\right)_{\infty}}
$$

and

$$
s_{n}(z) \sim q^{-n / 2}\left(\bar{t}_{1}\right)^{n} z^{n} \frac{\left(\bar{t}_{1}, \bar{b} q, \bar{a} q^{1 / 2} / z, q^{-1 / 2} \bar{t}_{2} / z ; q\right)_{\infty}}{\left(q^{1 / 2} / z, \bar{b} q^{1 / 2} \bar{t}_{1} z, \bar{a} \bar{t}_{1}, \bar{t}_{1} \bar{t}_{2} q^{-1} ; q\right)_{\infty}}
$$

Therefore,

$$
\begin{aligned}
& r_{n}(z) \bar{s}_{n}(1 / z) \\
& \quad \sim\left(t_{1} t_{2} q^{-1}\right)^{n} \frac{\left(t_{1}, t_{2}, a q, b q, a q^{1 / 2} z, b q^{1 / 2} / z, q^{-1 / 2} t_{1} / z, q^{-1 / 2} t_{2} z ; q\right)_{\infty}}{\left(a t_{1}, b t_{2}, t_{1} t_{2} q^{-1}, t_{1} t_{2} q^{-1}, a q^{1 / 2} t_{2} z, b q^{1 / 2} t_{1} / z, q^{1 / 2} z, q^{1 / 2} / z ; q\right)_{\infty}}
\end{aligned}
$$


In terms of the corresponding biorthogonal sets $\left\{R_{n}(z)\right\}$ and $\left\{S_{n}(z)\right\}$ (3.15)

$$
R_{n}(z)=R_{n}\left(z, a, b, t_{1}, t_{2}\right):=t_{2}^{-n} q^{n / 2}\left\{\frac{\left(a b t_{1} t_{2}\right)_{2 n}\left(t_{1} t_{2} q^{-1}\right)_{n}}{(q)_{n}(a b q)_{n}\left(a b t_{1} t_{2} q^{n-1}\right)_{n}}\right\}^{1 / 2} r_{n}(z)
$$

and

$$
S_{n}(z)=S_{n}\left(z, a, b, t_{1}, t_{2}\right):=R_{n}\left(z, \bar{b}, \bar{a}, \bar{t}_{2}, \bar{t}_{1}\right) .
$$

Relationships (3.12) and (3.13) lead to

Theorem 4. As $n \rightarrow \infty$ and for $\left|q^{1 / 2}\right|<|z|<\left|q^{-1 / 2}\right|$ we have

$$
R_{n}(z) \bar{S}_{n}(1 / z) \sim 1 / K(z) .
$$

We also note that $z^{-n} R_{n}(z)$ converges uniformly on compact subsets of $|z|>\left|q^{1 / 2}\right|$ to

$$
\begin{aligned}
\rho(z) & =\rho\left(z, a, b, t_{1}, t_{2}\right) \\
& =\frac{\left(t_{2}, a q, q^{1 / 2} b / z, q^{-1 / 2} t_{1} / z ; q\right)_{\infty}}{\left(b t_{2}, q^{1 / 2} / z, a q^{1 / 2} t_{2} z ; q\right)_{\infty}}\left\{\frac{\left(a b t_{1} t_{2}\right)_{\infty}}{\left(q, a b q, t_{1} t_{2} q^{-1} ; q\right)_{\infty}}\right\}^{1 / 2},
\end{aligned}
$$

and, using (3.18) and (3.16), we see $z^{-n} S_{n}(z)$ converges to

$$
\sigma(z)=\sigma\left(z, a, b, t_{1}, t_{2}\right)=\rho\left(z, \bar{b}, \bar{a}, \bar{t}_{2}, \bar{t}_{1}\right) .
$$

This takes us to

Theorem 5. We have

$$
\lim _{r \rightarrow 1+} \rho\left(r e^{i \theta}\right) \overline{\sigma\left(r e^{i \theta}\right)}=1 / K\left(e^{i \theta}\right) .
$$

\section{EPILOG}

The asymptotics of polynomials orthogonal on a compact set contain a wealth of spectral information on the spectrum and the spectral measures of the polynomials. For example, under certain conditions (see Szegö [20] and Grenander and Szegö [8]), a polynomial system $\left\{S_{n}(x)\right\}$ orthonormal on $[-1,1]$ will have the asymptotic development

$$
S_{n}(x) \sim z^{-n} / D(z), \quad x \in C /[-1,1], z:=x-\sqrt{x^{2}-1}, \text { as } n \rightarrow \infty
$$

where $D(z)$ is analytic in the open unit disc. The weight function $w(x)$ is related to the function $D(z)$ by

$$
w(x)=\left(1-x^{2}\right)^{-1 / 2} \lim _{r \rightarrow 1-} D\left(r e^{i \theta}\right) D\left(r e^{-i \theta}\right) .
$$

For polynomial sets, $\left\{\phi_{n}(z)\right\}$, orthogonal on the unit circle (see $[8$, p. 51]) we have

$$
\lim _{n \rightarrow \infty} z^{-n} \phi_{n}(z)=1 / \bar{g}(1 / z), \quad|z|>1
$$


where (see $[8$, p. 25$])$ almost everywhere on $[0,2 \pi]$ we have

$$
\lim _{r \rightarrow 1-}\left|g\left(r e^{i \theta}\right)\right|^{2}=w(\theta)=\alpha^{\prime}(\theta)
$$

where $\alpha(\theta)$ is the measure with respect to which $\left\{\phi_{n}(z)\right\}$ are orthogonal.

Szegö introduced his theory of polynomial sets orthogonal on the unit circle in the early twenties. He assumed that the orthogonality measure is a positive probability measure on the circle with infinite support. His assumption is equivalent to positivity of the associated Toeplitz determinants. Under these conditions the trigonometric moment problem has a unique solution. Many authors contributed to Szegö's theory, and it is now considered to be a fairly mature theory. To the best of our knowledge there is no such general theory known for biorthogonal rational functions on the unit circle. Part of the difficulty is that the measure with respect to which the functions are orthogonal may not be unique, and results as precise as those that exist for orthogonal polynomials on an interval or orthogonal polynomials on the unit circle cannot, in general, be expected.

Despite this, and as we have seen above, certain properties seem to carry over. The asymptotic and limiting relations obtained in the last section are not predicted by any theory that we know. Yet they suggest that Szegö's theory may be contained in a more general theory for rational functions biorthogonal on the unit circle. Even more surprising are the asymptotic relations (3.17) and (3.19) which seem to give a mechanism for recovering the weight function from the asymptotics of the rational biorthogonal functions.

\section{ACKNOWLEDGMENT}

The authors wish to thank Professor M. Rahman for some valuable advise which they received from him during the preparation of this manuscript.

\section{REFERENCES}

1. W. A. Al-Salam and A. Verma, q-analogs of some biorthogonal functions, Canad. Math. Bull. 26 (1983), 225-227.

2. R. Askey and J. Wilson, Some basic hypergeometric polynomials that generalize Jacobi polynomials, Mem. Amer. Math. Soc., No. 319, Amer. Math. Soc., Providence, RI, 1985.

3. G. Baxter, Polynomials defined by a difference system, J. Math. Anal. Appl. 2 (1961), 223-263.

4. A convergence equivalence related to polynomials orthogonal on the unit circle, Trans. Amer. Math. Soc. 99 (1961), 471-487.

5. C. Brezinski, Biorthogonality and its applications to numerical analysis, Marcel Dekker, New York, 1992.

6. G. Gasper and M. Rahman, Basic hypergeometric series, Cambridge Univ. Press, Cambridge, 1990.

7. L. Ya. Geronimus, Orthogonal polynomials, Consultant Bureau, New York, 1961.

8. U. Grenander and G. Szegö, Toeplitz forms and their applications, Univ. of California Press, Berkeley and Los Angeles, 1958.

9. W. Hahn, Beiträge zur Theorie der Heineschen Reihen, Math. Nachr. 2 (1949), 263-278.

10. A. Iserles and Norsett, On the theory of biorthogonal polynomials, Trans. Amer. Math. Soc. 306 (1988), 455-474.

11. M. E. H. Ismail and D. Masson, Q-Hermite polynomials, biorthogonal rational functions, and $q$-beta intergals (to appear). 
12. M. E. H. Ismail and D. Stanton, On the Askey-Wilson and Rogers polynomials, Canad. J. Math. 40 (1988), 1025-1045.

13. M. E. H. Ismail and J. Wilson, Asymptotic and generating relations for the q-Jacobi and ${ }_{4} \phi_{3}$ polynomials, Approx. Theory 36 (1982), 43-54.

14. P. I. Pastro, Orthogonal polynomials and some q-beta integrals of Ramanujan, J. Math. Anal. Appl. 112 (1982), 517-540.

15. M. Rahman, An integral representation of $a_{10} \phi_{9}$ and continuous biorthogonal ${ }_{10} \phi_{9}$ rational functions, Canad. J. Math. 38 (1986), 605-618.

16. Biorthogonality of a system of rational functions with respect to a positive measure on [-1, 1], SIAM J. Math. Anal. 22 (1991), 1421-1431.

17. G. Szegö, Ein Beitrag zur Theorie der Thetafunktionen, Sitz. Preuss. Akad. Wiss. Phys. Math. Kl. XIX (1926), 242-252; reprinted in Collected papers, Vol. I, Birkhäuser, Boston, MA,(, 1982).

18. _ Collected papers (R. Askey, ed.), Birkhäuser-Verlag, Boston, MA, 1982.

19. __ Beiträge zu Theorie der Toeplitzchen Formen. II, Math. Z. 9 (1982), 167-190; reprinted in Collected papers, Vol. I, Birkhäuser, Boston, MA, 1982.

20. __ Orthogonal polynomials, fourth ed., Amer. Math. Soc., Providence, RI, 1975.

Department of Mathematics, University of Alberta, Edmonton, Canada T6G 2G1

E-mail address: waleed@euler.math.ualberta.ca

Department of Mathematics, University of South Florida, Tampa, Florida 33620-5700

E-mail address: ismail@math.usf.edu 